\title{
Trajectories of Waist-to-Hip Ratio and Adverse Outcomes in Heart Failure with Mid-Range Ejection Fraction
}

\author{
Feng Gao ${ }^{a}$ Jindong Wan ${ }^{b, c}$ Banglong $X^{a} \quad$ Xiaochen Wang ${ }^{a}$ \\ Xianhe Lin ${ }^{d}$ Peijian Wang ${ }^{b, c}$
}

${ }^{a}$ Department of Cardiology, The Second Affiliated Hospital of Anhui Medical University, Hefei, PR China; ${ }^{b}$ Department of Cardiology, The First Affiliated Hospital of Chengdu Medical College, Chengdu, PR China; ' ${ }^{C}$ Key Laboratory of Aging and Vascular Homeostasis of Sichuan Higher Education Institutes, Chengdu, PR China; ${ }^{\mathrm{d}}$ Department of Cardiology, The First Affiliated Hospital of Anhui Medical University, Hefei, PR China

\section{Keywords}

Waist-to-hip ratio $\cdot$ Heart failure with mid-range ejection fraction - Trajectory · Prognosis

\begin{abstract}
Background: Waist-to-hip ratio (WHR) is a strong predictor of mortality in patients with heart failure (HF). However, common WHR trajectories are not well established in HF with mid-range ejection fraction (HFmrEF) persons, and their relationship to clinical outcomes remains uncertain. Method: We prospectively enrolled 1,396 participants with HFmrEF (left ventricular ejection fraction 40-49\%) from April 2013 through April 2017. The waist and hip circumferences of the subjects were measured at regular intervals, and the WHR was calculated as waist circumference divided by hip circumference. Latent mixture modeling was performed to identify WHR trajectories. We then used Cox proportional-hazard models to examine the association between WHR trajectory patterns and incident HF, incident cardiovascular disease (CVD), and all-cause mortality. Results: We identified four distinct WHR trajectory patterns: leanmoderate increase $(9.2 \%)$, medium-stable/increase (32.7\%), heavy-stable/increase (48.0\%), and heavy-moderate decrease (10.1\%). After multivariable adjustment, the heavy-stable/increase and heavy-moderate decrease patterns were associated with an increased all-cause mortality risk (heavy-stable/increase: adjusted hazard ratio [HR] 3.18, 95\% confidence interval [CI] 2.75-4.62; heavy-moderate decrease: adjusted HR 2.32, 95\% Cl 1.71-3.04), incident CVD risk (heavy-stable/increase: adjusted HR 4.03, 95\% Cl 2.39-4.91; heavy-moderate decrease:
\end{abstract}

Feng Gao and Jindong Wan contributed equally to this work.

Peijian Wang

Department of Cardiology, The First Affiliated Hospital of

Chengdu Medical College, 278 Baoguang Avenue, Xindu District

Chengdu, Sichuan 610500 (PR China)

wpjmed@ aliyun.com

\section{Xianhe Lin}

Department of Cardiology

The First Affiliated Hospital of Anhui Medical University Hefei, Anhui 230022 (PR China)

xianhelin@sina.com 
adjusted HR 3.05, 95\% Cl 2.34-4.09), and incident HF risk (heavy-stable/increase: adjusted HR 2.72, 95\% Cl 2.05-3.28; heavy-moderate decrease: adjusted HR 2.39, 95\% Cl 1.80-3.03) with reference to the lean-moderate increase pattern. Conclusion: Among patients with HFmrEF, the trajectories of WHR gain are associated with poor outcomes. These findings highlight the importance of abdominal fat accumulation management during the progression of HFmrEF.

(C) 2020 The Author(s)

Published by S. Karger AG, Basel

\section{Introduction}

Heart failure (HF) with mid-range ejection fraction (HFmrEF) is a transitional status between HF with preserved ejection fraction (HFpEF) and HF with reduced ejection fraction (HFrEF) [1, 2]. Patients with HFpEF have a poor prognosis similar to those with $\operatorname{HFrEF}[3,4]$. Identifying and screening modifiable risk factors for development of HFmrEF are critical to design effective prevention programs, as recommended by the guidelines [5].

Obesity is a known risk factor for the progression of HF [6]; however, a large body of evidence from epidemiologic studies has shown an obesity paradox, i.e., a higher body mass index (BMI) predicts a lower risk of death in patients with HF [7-9]. The precise pathophysiological mechanisms regarding this paradox remain uncertain. The BMI is the most common index to define obesity, whereas abdominal fat accumulation is not well described $[10,11]$. Moreover, subjects with a high BMI may be misclassified as having HF because of dyspnea [12]. Particularly, abdominal fat has been confirmed as a risk predictor of HF, and is known to predict mortality in individuals with coronary artery disease or even in the general population $[13,14]$. The commonly used measurement of abdominal fat is the waist-to-hip ratio (WHR) $[13,15,16]$. Of note, prior studies were based on a single WHR measure, failing to account for the potential effect of change in WHR values over time $[13,17,18]$. Furthermore, whether patterns of WHR change over time can predict clinical outcome has not been studied in populations with HFmrEF.

In this study, we assessed the association between WHR trajectory patterns and subsequent outcomes in patients with established HFmrEF.

\section{Subjects and Methods}

\section{Study Population}

Consecutive patients with a newly diagnosed HF were prospectively enrolled from April 2013 to April 2017 in the Second Hospital of Anhui Medical University and the First Affiliated Hospital of Chengdu Medical College. Initially eligible patients included those aged $\geq 18$ years who complied with the HF criteria (signs and symptoms), with a left ventricular ejection fraction $\geq 40 \%$, and a B-type natriuretic peptide (BNP) level $>35 \mathrm{pg} / \mathrm{mL}$ [1]. Patients who had chronic renal failure, hemodialysis, absence of waist circumference and hip circumference measures, systemic inflammatory disease, peripheral artery disease, a BMI $<18.5 \mathrm{~kg} / \mathrm{m}^{2}$, or an unknown follow-up status were excluded. Briefly, the remaining 1,396 patients who had an ascertained vital status were finally included (Fig. 1A).

\section{Data Collection}

The detailed medical histories and relevant baseline clinical assessments (including baseline demographics and laboratory results) of each patient were recorded. All monthly assessments of waist and hip circumference were performed thrice by trained medical technologists in accordance with a standardized protocol described elsewhere [13, 19], and the

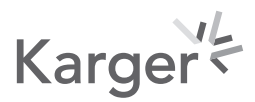


A

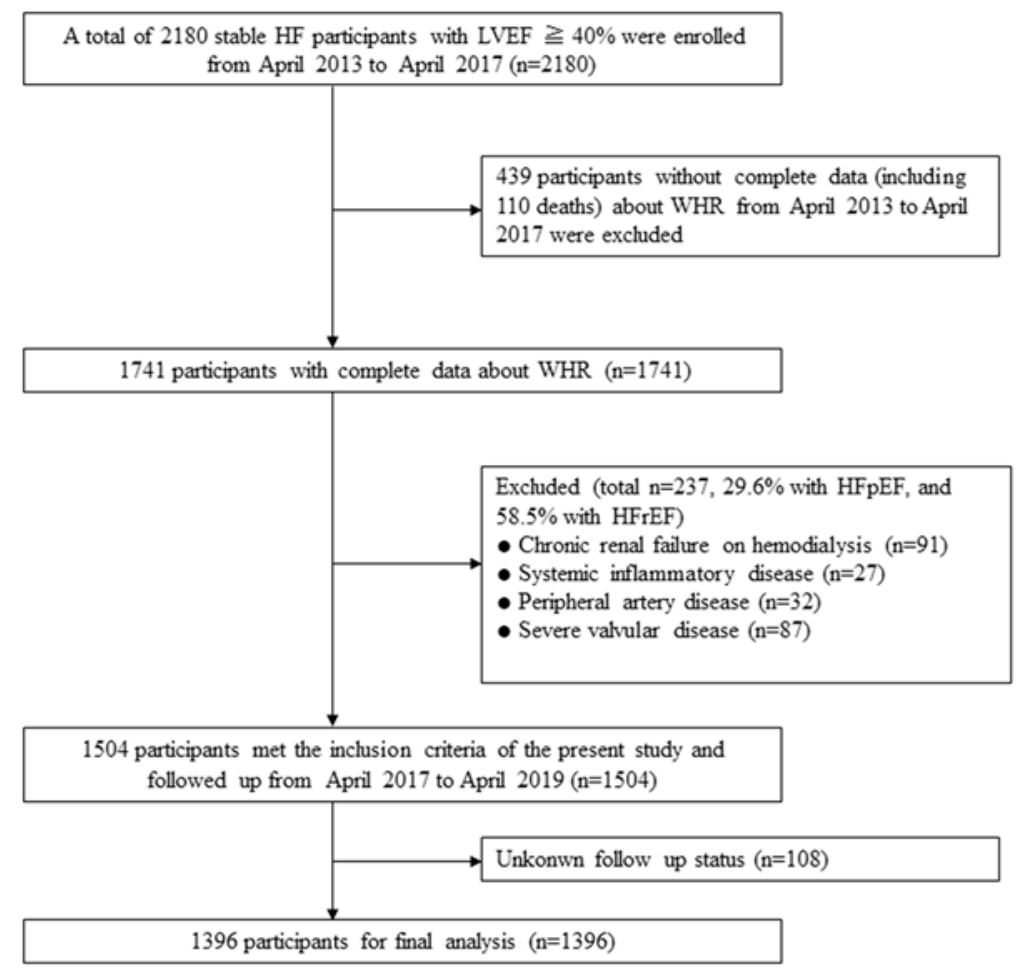

$\mathrm{B}$

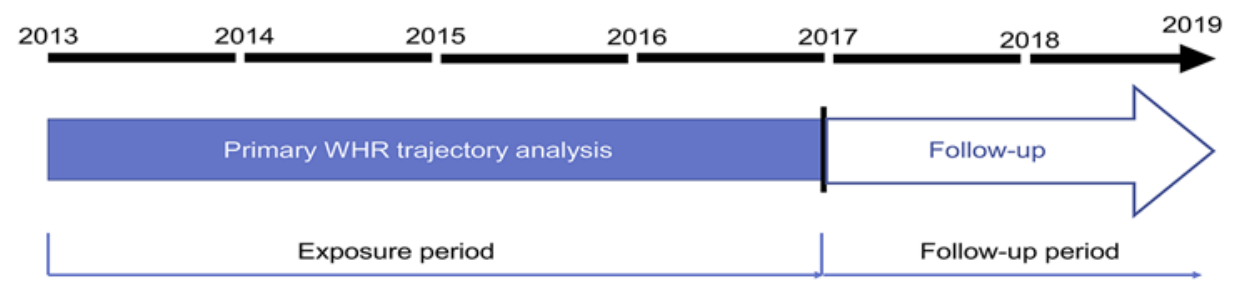

Fig. 1. A Flowchart showing the enrollment protocol. B Timeline of exposure and follow-up assessment. HF, heart failure; HFmrEF, HF with mid-range ejection fraction; HFpEF, HF with preserved ejection fraction; HFrEF, HF with reduced ejection fraction; LVEF, left ventricular ejection fraction; WHR, waist-to-hip ratio.

average result was recorded. Waist circumference divided by hip circumference defined the WHR $[13,20]$. A high WHR was $\geq 0.90$ for men and $\geq 0.85$ for women $[13,19,20]$. We evaluated the body composition parameters yearly, using an automated body composition scan (Karada Scan HBF-701; Omron, Tokyo, Japan).

Immediately after the measurement of waist and hip circumference, images were acquired from stable patients using echocardiography (Vivid 9; GE Healthcare, Waukesha, 
WI, USA). All images and measurements were performed by two experienced cardiac sonographers with no knowledge of the study data. The left ventricular ejection fraction, left ventricular diameter at the end of diastole, and left ventricular diameter at the end of systole were measured according to the current guideline [1,21,22].

$\mathrm{HF}$ patients were categorized into HFpEF ( $\mathrm{EF} \geq 50 \%$ ) and HFrEF (EF <40\%) [1]. Patients with an EF of 40-49\% were considered HFmrEF [1]. Weight and WHR were evaluated after hemodynamic optimization in hypervolemic patients to avoid the potential confounding effects of edema $[23,24]$. The differentiation between abdominal fat accumulation and HF-related ascites (or visceral congestion) was made based on clinical assessment (comprehensive physical examination), serum BNP measurement, and abdominal ultrasound [25]. During each monthly follow-up visit, a physical examination was performed to evaluate the hemodynamic state. If visceral congestion and/or ascites were suspicious, a BNP measurement and abdominal ultrasound were performed.

\section{Outcomes}

The participants were regularly followed up by phone calls, mobile social media software, or clinical visits ( $\geq 4$ times a year) from April 2017 until April 2019 (Fig. 1B). The primary outcomes were a composite of incident HF, incident cardiovascular disease (CVD), and all-cause mortality. Incident HF was hospitalization for congestive HF and was defined as having been admitted with symptomatic congestive HF and actively being on intravenous drug administration for HF according to objective signs of worsening HF [26]. An incident HF diagnosis was carried out by a specialist and was based on clinical assessment, chest X-ray, and echocardiography [26]. Incident CVD was defined as the composite of coronary revascularization, unstable angina pectoris, and nonfatal myocardial infarction [27]. During the 2-year follow-up, all subjects were administered the Minnesota Living with Heart Failure Questionnaire (MLHFQ) and the Kansas City Cardiomyopathy Questionnaire (KCCQ), which are two widely used disease-specific quality-of-life measures for patients with HF $[28,29]$. Higher MLHFQ scores and lower KCCQ scores reflect worse quality of life, and the questions involve HF-related symptoms and signs $[28,29]$.

\section{Statistical Analysis}

Clinical data were summarized as percentages for categorical variables and as the mean \pm standard deviation (SD) for continuous variables. The sample size was calculated based on previous studies $[27,30]$ and $80 \%$ power. Comparisons between all variables from multiple groups were assessed by the $\chi^{2}$ test, Kruskal-Wallis test, or one-way ANOVA. To identify WHR trajectories, we analyzed the WHR records for the same participants. On the basis of the WHR records, a latent mixture modeling (PROC TRAJ) was built for identifying subgroups, which assessed by Bayesian information criterion for model fit [31, 32]. The average posterior probability of each pattern of WHR was $0.91,0.97,0.85$, and 0.87 , reflecting a good fit of trajectory assignment.

The study population was stratified into four distinct WHR trajectory patterns: leanmoderate increase (0.78-0.84), medium-stable/increase (0.86-0.88), heavy-stable/increase (0.90-0.98), and heavy-moderate decrease (1.02-0.87). Cumulative event rates were reported using Kaplan-Meier estimates. Hazard ratios (HRs) and 95\% confidence intervals (CIs) for cardiovascular events were assessed using Cox proportional-hazard models. Several sensitivity analyses were performed. The level of significance was set at $p<0.05$. SPSS (version 22.0; IBM Corp., Armonk, NY, USA) and STATA (version 12.0; Stata Corp., College Station, TX, USA) were used for statistical analyses.

\section{Karger'}


Fig. 2. Trajectories of WHR. The solid lines are estimated values, and the dotted lines show 95\% confidence intervals. WHR, waistto-hip ratio.

Gao et al.: WHR and HF with Mid-Range Ejection Fraction
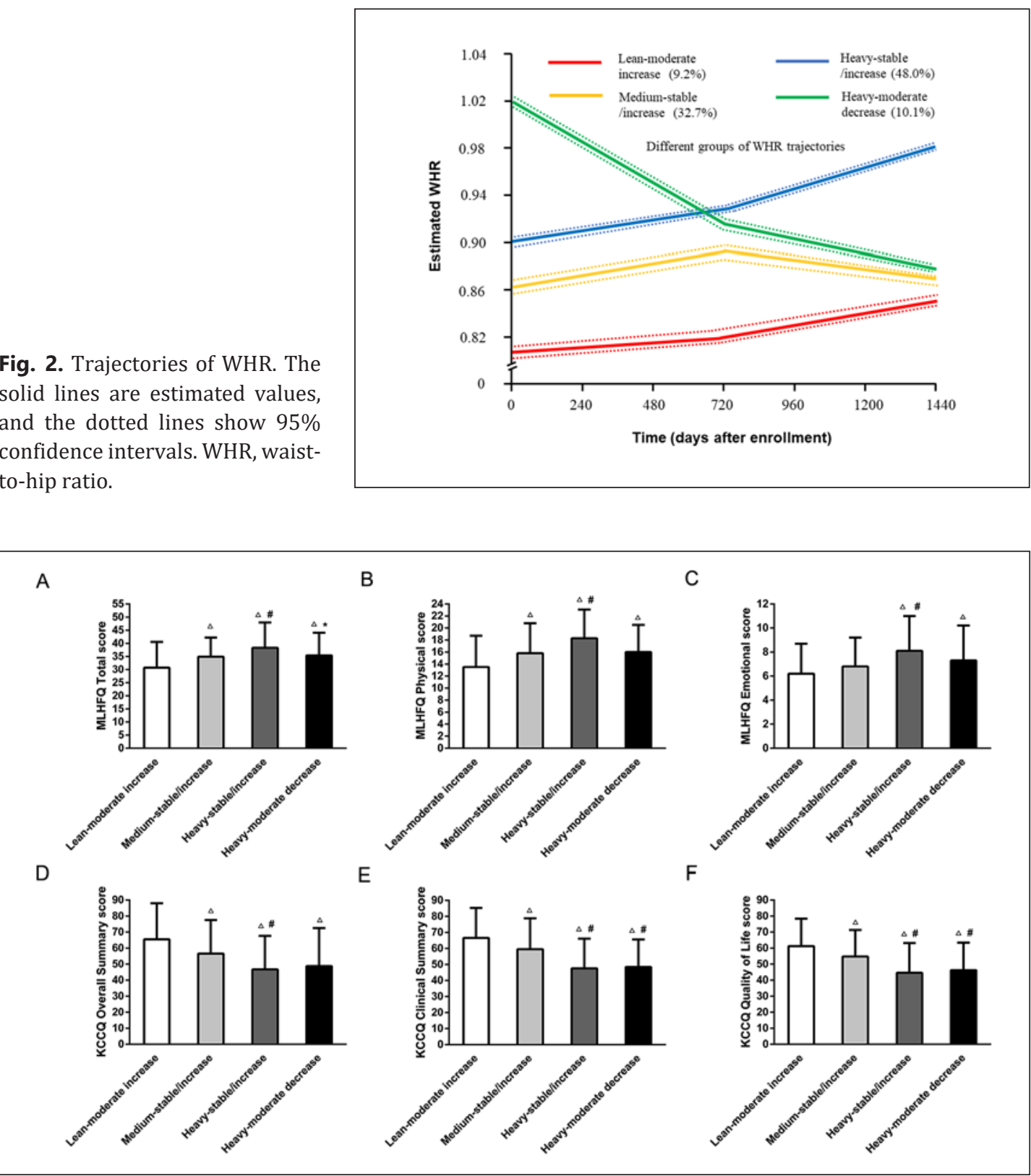

Fig. 3. A-F Quality of life in patients with heart failure with mid-range ejection fraction according to waistto-hip ratio trajectories. MLHFQ, Minnesota Living with Heart Failure Questionnaire; KCCQ, Kansas City Cardiomyopathy Questionnaire. ${ }^{\triangle} p<0.05$ compared with the lean-moderate increase group; ${ }^{\#} p<0.05$ compared with the medium-stable/increase group; ${ }^{*} p<0.05$ compared with the heavy-stable/increase group.

\section{Results}

\section{Baseline Characteristics}

The four distinct WHR trajectory patterns are depicted in Figure 2. During the period of 2013-2017, 9.2\% ( $n=129)$ of the individuals were categorized as having the lean-moderate increase pattern, 32.7\% $(n=456)$ the medium-stable/increase pattern, $48.0 \%(n=670)$ the heavy-stable/increase pattern, and $10.1 \%(n=141)$ the heavy-moderate decrease pattern. Table 1 displays the baseline characteristics of the 1,396 subjects. Compared with the other 


\begin{tabular}{l|l}
\hline Obes Facts 2020;13:344-357 \\
\hline DOI: $10.1159 / 000507708$ & $\begin{array}{l}\text { (C) 2020 The Author(s). Published by S. Karger AG, Basel } \\
\text { www.karger.com/ofa }\end{array}$ \\
\hline
\end{tabular}

Table 1. Patient characteristics in April 2017

\begin{tabular}{|c|c|c|c|c|c|}
\hline Parameter & $\begin{array}{l}\text { Lean-moderate } \\
\text { increase } \\
(n=129)\end{array}$ & $\begin{array}{l}\text { Medium-stable/ } \\
\text { increase } \\
(n=456)\end{array}$ & $\begin{array}{l}\text { Heavy-stable/ } \\
\text { increase } \\
(n=670)\end{array}$ & $\begin{array}{l}\text { Heavy-moderate } \\
\text { decrease } \\
(n=141)\end{array}$ & $\begin{array}{l}p \\
\text { value }\end{array}$ \\
\hline Age, years & $42.5 \pm 11.15$ & $44.7 \pm 11.52$ & $46.9 \pm 11.60$ & $44.3 \pm 11.37$ & $<0.001$ \\
\hline Men, $n(\%)$ & $24(18.6)$ & $240(52.6)$ & $549(81.9)$ & $72(51.1)$ & $<0.001$ \\
\hline College/university, $n(\%)$ & $14(10.9)$ & $39(8.6)$ & $34(5.1)$ & $10(7.1)$ & 0.037 \\
\hline Never-smokers, $n(\%)$ & $91(70.5)$ & $324(71.1)$ & $478(71.3)$ & $103(73.0)$ & 0.967 \\
\hline Alcohol intake, $n(\%)$ & $41(31.8)$ & $169(37.1)$ & $310(46.3)$ & $48(34.0)$ & $<0.001$ \\
\hline Physical activity $\geq 3$ times/week, $n(\%)$ & $38(29.5)$ & $123(27.0)$ & $201(30.0)$ & $44(31.2)$ & 0.666 \\
\hline \multicolumn{6}{|l|}{ NYHA class, $n(\%)$} \\
\hline I & $42(32.6)$ & $141(30.9)$ & $201(30.0)$ & $44(31.2)$ & 0.936 \\
\hline II & $72(55.8)$ & $253(55.5)$ & $369(55.1)$ & $78(55.3)$ & 0.997 \\
\hline III & $15(11.6)$ & $62(13.6)$ & $100(14.9)$ & $19(13.5)$ & 0.761 \\
\hline BMI, $\mathrm{kg} / \mathrm{m}^{2}$ & $22.2 \pm 3.02$ & $24.3 \pm 3.16$ & $26.0 \pm 3.50$ & $24.7 \pm 3.68$ & $<0.001$ \\
\hline WHR (April 2013) & $0.78 \pm 0.06$ & $0.86 \pm 0.07$ & $0.90 \pm 0.03$ & $1.02 \pm 0.04$ & $<0.001$ \\
\hline WHR (April 2017) & $0.84 \pm 0.03$ & $0.88 \pm 0.05$ & $0.95 \pm 0.06$ & $0.86 \pm 0.06$ & $<0.001$ \\
\hline Heart rate, bpm & $71.2 \pm 10.12$ & $73.6 \pm 9.59$ & $74.3 \pm 10.09$ & $73.1 \pm 9.82$ & $<0.001$ \\
\hline SBP, mm Hg & $116.8 \pm 18.74$ & $124.3 \pm 16.23$ & $129.2 \pm 19.75$ & $122.1 \pm 15.64$ & $<0.001$ \\
\hline DBP, mm Hg & $73.4 \pm 10.82$ & $81.5 \pm 11.70$ & $85.9 \pm 12.52$ & $78.7 \pm 9.63$ & $<0.001$ \\
\hline \multicolumn{6}{|l|}{ Comorbidity, $n(\%)$} \\
\hline Hypertension & $14(10.9)$ & $98(21.5)$ & $248(37.0)$ & $34(24.1)$ & $<0.001$ \\
\hline Diabetes mellitus & $6(4.7)$ & $46(10.1)$ & $120(17.9)$ & $16(11.3)$ & $<0.001$ \\
\hline Hyperlipidemia & $25(19.4)$ & $100(21.9)$ & $154(23.0)$ & $29(20.6)$ & 0.787 \\
\hline COPD & $21(16.3)$ & 87 (19.1) & $140(20.9)$ & $25(17.7)$ & 0.571 \\
\hline Prior PCI & $18(14.0)$ & $75(16.4)$ & $120(17.9)$ & $23(16.3)$ & 0.724 \\
\hline Atrial fibrillation & $19(14.7)$ & $77(16.9)$ & $123(18.4)$ & $22(15.6)$ & 0.692 \\
\hline Acute heart failure ( $\geq 3$ times/year) & $4(3.1)$ & $19(4.2)$ & $44(6.6)$ & $9(6.4)$ & 0.195 \\
\hline Infectious disease & $11(8.5)$ & $43(9.4)$ & $74(11.0)$ & $15(10.6)$ & 0.745 \\
\hline Peripheral edema present & $61(47.3)$ & $251(55.0)$ & $429(64.0)$ & $87(61.7)$ & 0.001 \\
\hline Rales present & $47(36.4)$ & $178(39.0)$ & $282(42.1)$ & $58(41.1)$ & 0.569 \\
\hline Elevated JVP & $32(24.8)$ & $124(27.2)$ & $175(26.1)$ & $40(28.4)$ & 0.896 \\
\hline \multicolumn{6}{|l|}{ Laboratory* } \\
\hline Fasting glucose, $\mathrm{mmol} / \mathrm{L}$ & $8.1 \pm 2.1$ & $8.2 \pm 1.4$ & $8.4 \pm 0.8$ & $8.2 \pm 1.9$ & 0.663 \\
\hline $\mathrm{TC}, \mathrm{mmol} / \mathrm{L}$ & $4.75 \pm 1.32$ & $5.01 \pm 1.28$ & $6.24 \pm 1.22$ & $5.10 \pm 1.30$ & $<0.001$ \\
\hline $\mathrm{TG}, \mathrm{mmol} / \mathrm{L}$ & $1.33 \pm 0.47$ & $1.71 \pm 0.51$ & $1.92 \pm 0.48$ & $1.75 \pm 0.52$ & $<0.001$ \\
\hline HDL-C, mmol/L & $1.66 \pm 0.48$ & $1.67 \pm 0.42$ & $1.63 \pm 0.38$ & $1.65 \pm 0.45$ & 0.922 \\
\hline LDL-C, mmol/L & $2.31 \pm 0.96$ & $2.45 \pm 0.90$ & $3.54 \pm 0.93$ & $1.92 \pm 0.78$ & $<0.001$ \\
\hline $\mathrm{BNP}, \mathrm{pg} / \mathrm{mL}$ & $195.4 \pm 105.62$ & $219.2 \pm 147.35$ & $227.6 \pm 164.43$ & $198.8 \pm 116.74$ & 0.013 \\
\hline $\mathrm{eGFR}, \mathrm{mL} / \mathrm{min} / 1.73 \mathrm{~m}^{2}$ & $87.2 \pm 9.81$ & $86.8 \pm 9.12$ & $75.4 \pm 9.37$ & $86.3 \pm 9.45$ & 0.032 \\
\hline Fat mass, $\% *$ & $29.9 \pm 7.5$ & $30.5 \pm 7.4$ & $32.3 \pm 7.3$ & $31.7 \pm 7.6$ & $<0.001$ \\
\hline SCF, $\% *$ & $20.8 \pm 7.9$ & $25.3 \pm 7.6$ & $26.4 \pm 7.5$ & $25.9 \pm 7.6$ & 0.003 \\
\hline $\mathrm{VF}^{*}$ & $8.6 \pm 5.1$ & $9.1 \pm 4.9$ & $9.9 \pm 5.3$ & $9.5 \pm 5.4$ & 0.015 \\
\hline FFM, \%* & $24.8 \pm 4.9$ & $24.7 \pm 5.1$ & $25.2 \pm 5.5$ & $24.9 \pm 4.7$ & 0.370 \\
\hline LVEF, \%* & $46 \pm 3.5$ & $44 \pm 2.7$ & $43 \pm 2.5$ & $45 \pm 4.1$ & 0.350 \\
\hline \multicolumn{6}{|l|}{ Change in LVEF (increase/decrease } \\
\hline$\geq 10 \%), n(\%)$ & $17(13.2)$ & $69(15.1)$ & $128(19.1)$ & $25(17.7)$ & 0.205 \\
\hline LVDd, mm* & $51.3 \pm 8.5$ & $53.4 \pm 7.8$ & $55.7 \pm 8.6$ & $53.9 \pm 7.5$ & 0.211 \\
\hline LVDs, mm* & $42.5 \pm 6.1$ & $44.8 \pm 5.9$ & $45.7 \pm 6.2$ & $44.5 \pm 6.4$ & 0.683 \\
\hline \multicolumn{6}{|l|}{ Medication, $n(\%)$} \\
\hline ACE-I/ARB & $88(68.2)$ & 337 (73.9) & $504(75.2)$ & $100(70.9)$ & 0.333 \\
\hline Spironolactone & $38(29.5)$ & $141(30.9)$ & 227 (33.9) & $45(31.9)$ & 0.649 \\
\hline Beta-blocker & $82(63.6)$ & $323(70.8)$ & $489(73.0)$ & $95(67.4)$ & 0.179 \\
\hline Diuretics & $125(96.9)$ & 446 (97.8) & 657 (98.1) & $138(97.9)$ & 0.874 \\
\hline Statin & $72(55.8)$ & $264(57.9)$ & $408(60.9)$ & $83(58.9)$ & 0.629 \\
\hline \multicolumn{6}{|l|}{ Change in medication, $n(\%)$} \\
\hline Antihypertensive & $11(8.5)$ & $83(18.2)$ & $222(33.1)$ & $29(20.6)$ & $<0.001$ \\
\hline Hypoglycemic & $4(3.1)$ & $37(8.1)$ & $94(14.0)$ & $13(9.2)$ & $<0.001$ \\
\hline Lipid-lowering drugs & $14(10.9)$ & $50(11.0)$ & $82(12.2)$ & $19(13.5)$ & 0.821 \\
\hline
\end{tabular}




\section{Footnote to Table 1}

Values are mean \pm SD or $n(\%)$. A $p$ value of $<0.05$ was considered statistically significant. NYHA, New York Heart Association; BMI, body mass index; WHR, waist-to-hip ratio; SBP, systolic blood pressure; DBP, diastolic blood pressure; COPD, chronic obstructive pulmonary disease; PCI, percutaneous coronary intervention; JVP, jugular venous pressure; TC, total cholesterol; TG, triglycerides; HDL-C, high-density lipoprotein cholesterol; LDL-C, low-density lipoprotein cholesterol; BNP, B-type natriuretic peptide; eGFR, estimated glomerular filtration rate; SCF, subcutaneous fat; VF, visceral fat; FFM, fat-free mass; LVEF, left ventricular ejection fraction; LVDd, left ventricular diameter diastolic; LVDs, left ventricular diameter systolic; ACE-I, angiotensin-converting enzyme inhibitor; ARB, angiotensin-II receptor blocker. * Average levels based on 5 measurements (in 2013, 2014, 2015, 2016, and 2017).

Fig. 4. Kaplan-Meier analysis of all-cause mortality (A), incident CVD (B), and incident HF (C) in patients with HFmrEF according to WHR trajectories. CVD, cardiovascular disease; HF, heart failure; HFmrEF, HF with mid-range ejection fraction; WHR, waist-tohip ratio.
A

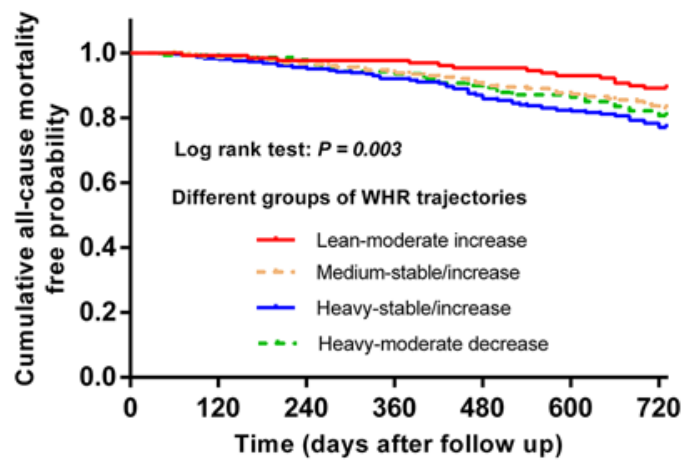

B

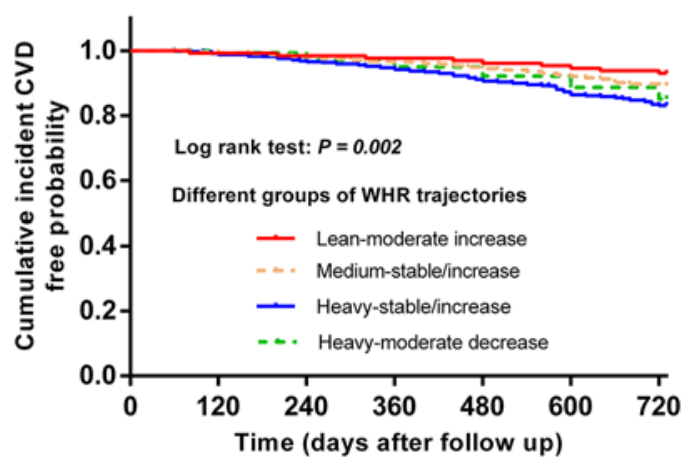

C

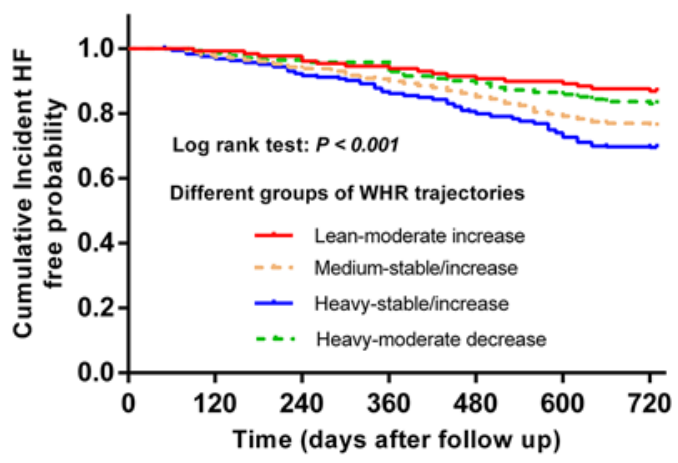


Gao et al.: WHR and HF with Mid-Range Ejection Fraction

Table 2. Association of WHR trajectories with outcomes

\begin{tabular}{|c|c|c|c|c|}
\hline & \multirow[t]{2}{*}{ Events } & \multirow{2}{*}{$\begin{array}{l}\text { Event rate } \\
\text { (per } 1,000 \\
\text { person-years) }\end{array}$} & \multirow{2}{*}{$\frac{\text { Demographics }^{a}}{\text { HR }(95 \% \mathrm{CI})}$} & \multirow{2}{*}{$\frac{\text { Demographics + WHR }}{\text { HR }(95 \% \mathrm{CI})}$} \\
\hline & & & & \\
\hline \multicolumn{5}{|l|}{ All-cause mortality } \\
\hline Lean-moderate increase $(n=129)$ & $13(10.1)$ & 11.2 & 1.00 (reference) & 1.00 (reference) \\
\hline Medium-stable/increase $(n=456)$ & $77(16.9)$ & 20.3 & $1.09(0.83-1.22)$ & $1.02(0.75-1.38)$ \\
\hline Heavy-stable/increase $(n=670)$ & $154(23.0)$ & 29.8 & $2.18(0.75-3.62)$ & $1.42(0.68-1.76)$ \\
\hline Heavy-moderate decrease $(n=141)$ & $27(19.1)$ & 23.7 & $2.32(0.71-3.04)$ & $1.48(0.91-1.89)$ \\
\hline \multicolumn{5}{|l|}{ Coronary-related events } \\
\hline Lean-moderate increase $(n=129)$ & $10(7.8)$ & 8.4 & 1.00 (reference) & 1.00 (reference) \\
\hline Medium-stable/increase $(n=456)$ & $50(11.0)$ & 12.3 & $1.21(0.66-1.78)$ & $1.49(0.93-2.19)$ \\
\hline Heavy-stable/increase $(n=670)$ & $113(16.9)$ & 20.3 & $1.24(0.95-1.61)$ & $1.35(0.87-1.95)$ \\
\hline Heavy-moderate decrease $(n=141)$ & $21(14.9)$ & 17.5 & $2.35(1.57-3.46)$ & $2.22(1.34-3.23)$ \\
\hline \multicolumn{5}{|l|}{ HF-related events } \\
\hline Lean-moderate increase $(n=129)$ & $17(13.2)$ & 15.2 & 1.00 (reference) & 1.00 (reference) \\
\hline Medium-stable/increase $(n=456)$ & $109(23.9)$ & 31.4 & $1.13(0.80-1.52)$ & $1.67(0.42-2.08)$ \\
\hline Heavy-stable/increase $(n=670)$ & $202(30.1)$ & 43.2 & $1.47(0.73-2.38)$ & $1.79(1.23-2.72)$ \\
\hline Heavy-moderate decrease $(n=141)$ & $24(17.0)$ & 20.5 & $1.91(0.65-3.27)$ & $1.53(1.04-2.26)$ \\
\hline
\end{tabular}

WHR, waist-to-hip ratio; HF, heart failure; HR, hazard ratio; CI, confidence interval. ${ }^{\text {a }}$ Age and sex. ${ }^{\text {b }}$ April 2017 WHR.

three WHR trajectory patterns, individuals with the heavy-stable/increase pattern were more likely (1) to be men, older, and alcohol drinkers; (2) to have a higher BMI, heart rate, systolic and diastolic blood pressure, total cholesterol, triglycerides, low-density lipoproteincholesterol, and BNP level; and (3) to have a higher prevalence of hypertension and diabetes mellitus. Moreover, they were less likely to have higher education and had a lower estimated glomerular filtration rate.

\section{Quality of Life}

All participants' quality of life values as assessed by the MLHFQ and KCCQ at the end of the 2-year follow-up are shown in Figure 3. Compared with the other three WHR trajectory patterns, the individuals with the heavy-stable/increase pattern had the highest score on the MLHFQ and the lowest score on the KCCQ.

\section{Outcomes during Follow-Up}

For the follow-up period from April 2017 through April 2019, the detailed clinical outcomes of interest are shown in Table 2 . The individuals with the heavy-stable/increase pattern had the highest risk of incident HF, incident CVD, and all-cause mortality. The survival curves of cumulative all-cause mortality, incident CVD, and incident HF for the four WHR trajectory groups are shown in Figure 4. As time went on, the WHR trajectory patterns were associated with all-cause mortality (log-rank test $p=0.003$; Fig. 4A), incident CVD (log-rank test $p=0.002$; Fig. 4B), and incident HF (log-rank test $p<0.001$; Fig. 4C).

\section{Cox Proportional-Hazard Analysis}

Table 2 shows the unadjusted cumulative incidence of all-cause mortality, incident CVD, and incident HF over time. After adjustment for potential confounders, the results of the multivariable analysis of all-cause mortality, incident CVD, and incident HF are shown in Figure 5. With reference to the lean-moderate increase pattern, the heavy-stable/increase pattern and the heavy-moderate decrease pattern were associated with an increased all- 
A

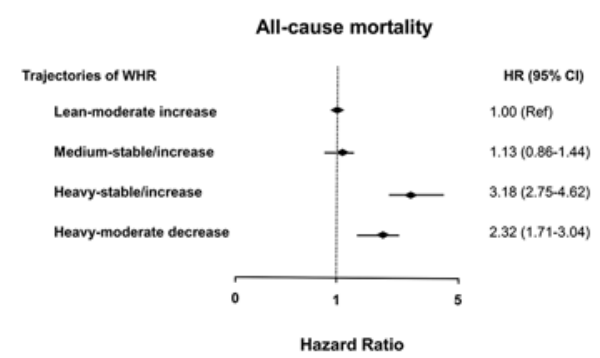

B

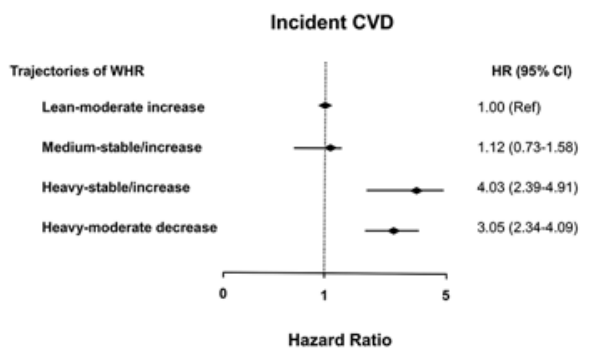

C

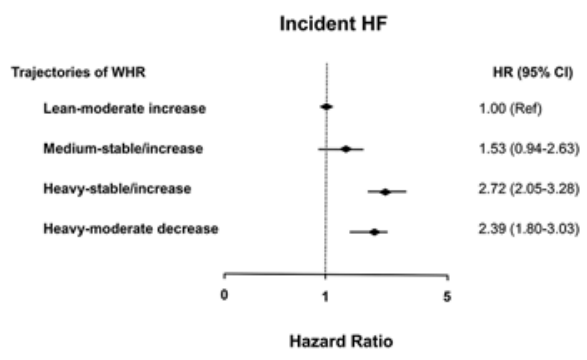

Fig. 5. Forest plot showing the results of multivariate Cox proportional-hazard analysis of all-cause mortality (A), incident CVD (B), and incident HF (C) in patients with HFmrEF according to WHR trajectories after adjustment for other confounders (hazard ratio $=1$ set at the reference of the lean-moderate increase group). Multivariate = demographics + WHR (April 2018 WHR) + cardiovascular risk factors (alcohol intake, body mass index, heart rate, systolic blood pressure, diastolic blood pressure, total cholesterol, triglycerides, low-density lipoprotein cholesterol, B-type natriuretic peptide, estimated glomerular filtration rate) + history of chronic medical conditions (hypertension, diabetes mellitus). CI, confidence interval; CVD, cardiovascular disease; HF, heart failure; HFmrEF, HF with mid-range ejection fraction; HR, hazard ratio; WHR, waist-to-hip ratio.

cause mortality risk (heavy-stable/increase pattern: adjusted HR 3.18, 95\% CI 2.75-4.62; heavy-moderate decrease pattern: adjusted HR 2.32, 95\% CI 1.71-3.04; Fig. 5A), incident CVD risk (heavy-stable/increase pattern: adjusted HR 4.03, 95\% CI 2.39-4.91; heavy-moderate decrease pattern: adjusted HR 3.05, 95\% CI 2.34-4.09; Fig. 5B), and incident HF risk (heavystable/increase pattern: adjusted HR 2.72, 95\% CI 2.05-3.28; heavy-moderate decrease pattern: adjusted HR 2.39, 95\% CI 1.80-3.03; Fig. 5C). Table 3 shows the sensitivity analyses after excluding some individuals. Whenever we excluded some subjects, in the following, the results revealed no obvious differences between these data and those described in Figure 5.

\section{Discussion}

This is the first study to demonstrate an association between higher WHR trajectory patterns (reflecting abdominal fat) and worse outcomes in patients with HFmrEF. Specifically, it provides a critical support for more expanded use of the WHR for abdominal obesity management in the setting of HFmrEF. 
Gao et al.: WHR and HF with Mid-Range Ejection Fraction

Table 3. Sensitivity analysis of hazard ratios and 95\% confidence intervals for outcomes according to the WHR trajectories

\begin{tabular}{|c|c|c|c|c|}
\hline & \multicolumn{4}{|c|}{ Hazard ratio ( $95 \%$ confidence interval) } \\
\hline & $\begin{array}{l}\text { lean-moderate } \\
\text { increase }\end{array}$ & $\begin{array}{l}\text { medium-stable/ } \\
\text { increase }\end{array}$ & $\begin{array}{l}\text { heavy-stable/ } \\
\text { increase }\end{array}$ & $\begin{array}{l}\text { heavy-moderate } \\
\text { decrease }\end{array}$ \\
\hline \multicolumn{5}{|c|}{ All-cause mortality } \\
\hline Model 1 & 1.00 (reference) & $1.31(1.07-1.58)$ & $1.52(1.13-1.89)$ & $1.65(1.30-2.11)$ \\
\hline Model 2 & 1.00 (reference) & $1.21(1.10-1.54)$ & $1.08(1.03-1.22)$ & $1.81(1.32-2.47)$ \\
\hline Model 3 & 1.00 (reference) & $1.39(1.07-1.95)$ & $1.62(1.30-2.71)$ & $1.66(1.22-2.89)$ \\
\hline Model 4 & 1.00 (reference) & $1.28(1.06-1.56)$ & $1.54(1.13-2.64)$ & $2.08(1.43-3.22)$ \\
\hline \multicolumn{5}{|c|}{ Coronary-related events } \\
\hline Model 1 & 1.00 (reference) & $1.56(1.15-2.23)$ & $1.29(1.07-2.32)$ & $1.55(1.26-2.78)$ \\
\hline Model 2 & 1.00 (reference) & $1.38(1.11-1.88)$ & $1.63(1.06-2.11)$ & $1.80(1.40-2.39)$ \\
\hline Model 3 & 1.00 (reference) & $1.33(1.18-1.79)$ & $1.75(1.30-3.47)$ & $1.83(1.27-2.86)$ \\
\hline Model 4 & 1.00 (reference) & $1.90(1.52-2.51)$ & $3.71(2.75-4.89)$ & $3.93(2.85-5.33)$ \\
\hline \multicolumn{5}{|c|}{ HF-related events } \\
\hline Model 1 & 1.00 (reference) & $2.18(1.67-2.83)$ & $3.16(1.69-4.03)$ & $4.20(3.71-4.95)$ \\
\hline Model 2 & 1.00 (reference) & $1.29(1.18-2.30)$ & $1.52(1.30-1.92)$ & 1.67 (1.27-1.99) \\
\hline Model 3 & 1.00 (reference) & $1.25(1.09-1.66)$ & $1.52(1.22-1.93)$ & $1.97(1.43-2.85)$ \\
\hline Model 4 & 1.00 (reference) & $3.12(1.95-6.50)$ & $8.10(5.32-10.20)$ & $9.17(4.88-14.33)$ \\
\hline
\end{tabular}

Model 1: we excluded the participants who had infectious disease, acute heart failure ( $\geq 3$ times/year); adjusted confounders are multivariate, which are the same as in Figure 5. Model 2: we excluded the participants who had diabetes mellitus at baseline or in the follow-up period; adjusted confounders are multivariate, which are the same as in Figure 5. Model 3: we excluded the participants who had a change in left ventricular ejection fraction (increase/decrease $\geq 10 \%$ ) between 2013 and 2017; adjusted confounders are multivariate, which are the same as in Figure 5. Model 4: we excluded the participants who had a change in medications (antihypertensive, hypoglycemic, and lipid-lowering drugs) between 2013 and 2017; adjusted confounders are multivariate, which are the same as in Figure 5. WHR, waist-to-hip ratio; HF, heart failure.

These findings provide unique insight into the role of WHR changes in patients with HFmrEF. A large number of studies have described that the prevalence of abdominal obesity defined by WHR measurement is increasing with age up to 60 years and is then followed by a decline $[33,34]$. By integrating the WHR into the modeling approach, we found unique WHR trajectory patterns in these patients with HFmrEF. In particular, we found that subjects who maintained a "heavy" trajectory had the highest mortality, whereas those who remained on a "lean" trajectory had the lowest risk of death. Previous studies have demonstrated that a higher WHR trajectory pattern was one of the predictors of all-cause mortality $[13,19]$. In a prospective cohort from Scotland, a higher WHR predicted a higher all-cause mortality risk in female patients with HF [13]; however, the authors did not adopt a trajectory modeling approach to minimize reverse causation. Two large prospective cohort studies using the BMI trajectory approach reported that individuals who gained weight in middle age or maintained a heavy body weight throughout life were at higher risk of mortality [35]; yet the authors did not focus on the association between abdominal fat assessed by the WHR and mortality among patients with HFmrEF. Furthermore, compared with those who maintained a "lean" trajectory, our results suggest that participants with a "heavy" trajectory throughout the HFmrEF settings had a substantially increased cardiovascular risk.

In addition, Tsujimoto and Kajio [24] recently added to the accumulating evidence that abdominal obesity measured via waist circumference significantly heightened cardiovascular mortality among subjects with HFpEF. In addition, a novel finding of our study is that a quality-of-life assessment of our subjects with HFmrEF revealed that a "heavy" trajectory was 
Gao et al.: WHR and HF with Mid-Range Ejection Fraction

significantly associated with a worse quality of life based on MLHFQ and KCCQ scores. Indeed, the impact of quality of life on HFmrEF has been shown to be strong in a retrospective analysis of clinical settings in China [36]; however, the authors did not account for the association between abdominal obesity assessed via the WHR and the psychosomatic state, which might reflect the efficacy of therapy.

Despite the well-recognized effect of abdominal obesity on the development of HF, there remains considerable uncertainty regarding the role of the WHR trajectory in cardiovascular events, due to the complex interaction of various cardiovascular risk factors. Several possible explanations have been proposed. First, patients with HFmrEF frequently suffer from multiple comorbidities, including hypertension, diabetes, obesity, coronary artery disease, and metabolic syndrome $[13,19,37]$. On account of these coexisting conditions, clinical trials have emphasized that an increase in WHR was a major contributor to a systemic proinflammatory state, which is involved in myocardial fibrosis, hypertrophy, and pathological remodeling, ultimately promoting left ventricular dysfunction $[13,38]$. As is known, abdominal obesity is strongly related to systemic inflammation, which is recognized to be associated with HF worsening $[39,40]$. Inflammation might have been responsible for the worse clinical outcome in participants with a "heavy" trajectory. Second, previous studies have shown that weight gain in middle life has also been related to several cardiometabolic abnormalities, such as insulin resistance, atherogenic dyslipidemia, hyperleptinemia, and low adiponectin levels $[24,35]$. Interestingly, even lean middle-aged individuals who had gained weight later had a higher risk of mortality. Results from prior BMI trajectory-based studies showed that HF in individuals with higher BMI levels was associated with a lower mortality risk, which is distinct from our results [35]. Tsujimoto and Kajio [24] revealed that HFpEF patients with higher waist circumference levels had higher mortality rates. These conflicting results may be due to the fact that the WHR is a better abdominal obesity measure than the BMI, possibly being able to evaluate abdominal fat more accurately. Third, obesity is often accompanied by psychosocial disorders, such as depression or anxiety, resulting in poor outcomes, especially in patients with HFmrEF [41, 42]. Previous studies have evaluated the association of weight loss with well-being outcomes in adults with HF $[41,42]$. Findings from epidemiologic studies indicate that abdominal obesity shares complex biologic, etiologic, and genetic substrates with psychosocial states of stress [42, 43]. The psychosocial state characterized by chronic hypercortisolism induces abdominal fat accumulation, depressive symptoms, and severely reduced quality of life [43].

\section{Strengths and Limitations}

The advantage of this study is that we repeated WHR measurements and evaluated the quality of life among all patients over a period of time. Significantly, the WHR measurement is fast, reliable, noninvasive, and cheap, making it a valuable measurement for clinical practice and lifestyle modification worldwide.

Our study, though, has several limitations. First, this is an observational study based on only two centers; a subgroup analysis considering men and women separately would be worth performing. Second, the WHR was measured by different medical staff members, even though they received standardized training. Third, the present study was conducted on a specific cohort of patients with HF but not on the general population. Hence, our results may not be applicable to other populations or geographic areas. Fourth, it is difficult to differentiate between fat and fluid, and the latter is significant in patients with HFmrEF. Fifth, normal values for the WHR might differ by ethnicity, and the follow-up period for the WHR trajectory was relatively short. Thus, longer follow-ups will be needed for other populations.

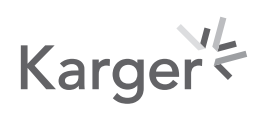




\section{Conclusions}

Among patients with HFmrEF, trajectories of WHR gain are significantly associated with poor outcomes. Recognition of the WHR trajectory patterns may lead to an early identification of high-risk patients with HFmrEF and optimization of strategies for long-term weight management. These findings highlight the importance of abdominal fat accumulation management across the progression of HFmrEF.

\section{Acknowledgments}

The authors would like to thank all the participants for their contribution to this study.

\section{Statement of Ethics}

The study was approved by the Institutional Review Board of the Second Hospital of Anhui Medical University and the First Affiliated Hospital of Chengdu Medical College and was conducted in accordance with the guidelines laid down in the Declaration of Helsinki. All patients provided informed consent.

\section{Disclosure Statement}

The authors have no conflicts of interest to declare.

\section{Funding Sources}

This research was supported by grants from the National Natural Science Foundation of China (81400289 and 81970262) (P.W.), the Program of Sichuan Youth Science and Technology Foundation in China (2016JQ0032) (P.W.), the Innovation Team Project Department of Education of Sichuan Province (18TD0030) (P.W.), and the Anhui Medical University Doctoral Startup Fund (2014BKJ038) (F.G.).

\section{Author Contributions}

P.W. and F.G. conceived and designed the experiments; all authors performed the experiments; J.W. and B.X. analyzed the data; J.W., B.X., and X.W. contributed reagents/materials/ analysis tools; P.W. and X.L. drafted and revised the paper. All authors read and approved the final manuscript.

\section{References}

1 Ponikowski P, Voors AA, Anker SD, Bueno H, Cleland JG, Coats AJ, et al.; ESC Scientific Document Group. 2016 ESC Guidelines for the diagnosis and treatment of acute and chronic heart failure: the Task Force for the diagnosis and treatment of acute and chronic heart failure of the European Society of Cardiology (ESC) Developed with the special contribution of the Heart Failure Association (HFA) of the ESC. Eur Heart J. 2016 Jul;37 (27): 2129-200. 


\begin{tabular}{|c|c|}
\hline Obes Facts 2020;13:344-357 & \\
\hline DOI: 10.1159/000507708 & $\begin{array}{l}\text { (c) } 2020 \text { The Author(s). Published by S. Karger AG, Basel } \\
\text { www.karger.com/ofa }\end{array}$ \\
\hline
\end{tabular}

Gao et al.: WHR and HF with Mid-Range Ejection Fraction

2 Gladden JD, Chaanine AH, Redfield MM. Heart Failure with Preserved Ejection Fraction. Annu Rev Med. 2018 Jan;69(29):65-79.

3 Ghio S, Raineri C, Scelsi L, Ašanin M, Polovina M, Seferovic P. Pulmonary hypertension and right ventricular remodeling in HFpEF and HFrEF. Heart Fail Rev. 2020 Jan;25(1):85-91.

4 Park JJ, Park CS, Mebazaa A, Oh IY, Park HA, Cho HJ, et al. Characteristics and outcomes of HFpEF with declining ejection fraction. Clin Res Cardiol. 2020 Feb;109(2):225-34.

5 van der Meer P, Gaggin HK, Dec GW. ACC/AHA versus ESC Guidelines on Heart Failure: JACC Guideline Comparison. J Am Coll Cardiol. 2019 Jun;73(21):2756-68.

6 Savji N, Meijers WC, Bartz TM, Bhambhani V, Cushman M, Nayor M, et al. The association of obesity and cardiometabolic traits with incident HFpEF and HFrEF. JACC Heart Fail. 2018 Aug;6(8):701-9.

7 Horwich TB, Fonarow GC, Clark AL. Obesity and the obesity paradox in heart failure. Prog Cardiovasc Dis. 2018 Jul-Aug;61(2):151-6.

8 Castleberry CD, Jefferies JL, Shi L, Wilkinson JD, Towbin JA, Harrison RW, et al. No obesity paradox in pediatric patients with dilated cardiomyopathy. JACC Heart Fail. 2018 Mar;6(3):222-30.

9 Powell-Wiley TM, Ngwa J, Kebede S, Lu D, Schulte PJ, Bhatt DL, et al. Impact of Body Mass Index on Heart Failure by Race/Ethnicity from the Get with the Guidelines-Heart Failure (GWTG-HF) Registry. JACC Heart Fail. 2018 Mar;6(3):233-42.

10 Edqvist J, Rawshani A, Adiels M, Björck L, Lind M, Svensson AM, et al. BMI, Mortality, and Cardiovascular Outcomes in Type 1 Diabetes: Findings against an Obesity Paradox. Diabetes Care. 2019 Jul;42(7):1297-304.

11 Samanta R, Pouliopoulos J, Kumar S, Narayan A, Nadri F, Qian P, et al. Influence of BMI on inducible ventricular tachycardia and mortality in patients with myocardial infarction and left ventricular dysfunction: the obesity paradox. Int J Cardiol. 2018 Aug;265(15):148-54.

12 Maron BJ, Rowin EJ, Udelson JE, Maron MS. Clinical spectrum and management of heart failure in hypertrophic cardiomyopathy. JACC Heart Fail. 2018 May;6(5):353-63.

13 Streng KW, Voors AA, Hillege HL, Anker SD, Cleland JG, Dickstein K, et al. Waist-to-hip ratio and mortality in heart failure. Eur J Heart Fail. 2018 Sep;20(9):1269-77.

14 Haykowsky MJ, Nicklas BJ, Brubaker PH, Hundley WG, Brinkley TE, Upadhya B, et al. Regional adipose distribution and its relationship to exercise intolerance in older obese patients who have heart failure with preserved ejection fraction. JACC Heart Fail. 2018 Aug;6(8):640-9.

15 Turcotte M, Abadi A, Peralta-Romero J, Suarez F, Reddon H, Gomez-Zamudio J, et al. Genetic contribution to waist-to-hip ratio in Mexican children and adolescents based on 12 loci validated in European adults. Int J Obes. 2019 Jan;43(1):13-22.

16 Lotta LA, Wittemans LB, Zuber V, Stewart ID, Sharp SJ, Luan J, et al. Association of genetic variants related to gluteofemoral vs abdominal fat distribution with type 2 diabetes, coronary disease, and cardiovascular risk factors. JAMA. 2018 Dec;320(24):2553-63.

17 Emdin CA, Khera AV, Natarajan P, Klarin D, Zekavat SM, Hsiao AJ, et al. Genetic association of waist-to-hip ratio with cardiometabolic traits, type 2 diabetes, and coronary heart disease. JAMA. 2017 Feb;317(6):626-34.

18 Morris A. Adipose tissue: apple or pear - waist-to-hip ratio and the risk of CHD and T2DM. Nat Rev Endocrinol. 2017 Apr;13(4):187-8.

19 Wan J, Zhou P, Wang D, Liu S, Yang Y, Hou J, et al. Impact of normal weight central obesity on clinical outcomes in male patients with premature acute coronary syndrome. Angiology. 2019 Nov;70(10):960-8.

20 Hamer M, Batty GD. Association of body mass index and waist-to-hip ratio with brain structure: UK Biobank study. Neurology. 2019 Feb;92(6):e594-600.

21 Lund LH, Claggett B, Liu J, Lam CS, Jhund PS, Rosano GM, et al. Heart failure with mid-range ejection fraction in CHARM: characteristics, outcomes and effect of candesartan across the entire ejection fraction spectrum. Eur J Heart Fail. 2018 Aug;20(8):1230-9.

22 Bhambhani V, Kizer JR, Lima JA, van der Harst P, Bahrami H, Nayor M, et al. Predictors and outcomes of heart failure with mid-range ejection fraction. Eur J Heart Fail. 2018 Apr;20(4):651-9.

23 Clark AL, Chyu J, Horwich TB. The obesity paradox in men versus women with systolic heart failure. Am J Cardiol. 2012 Jul;110(1):77-82.

24 Tsujimoto T, Kajio H. Abdominal obesity is associated with an increased risk of all-cause mortality in patients with HFpEF. J Am Coll Cardiol. 2017 Dec; 70(22):2739-49.

25 Thibodeau JT, Drazner MH. The role of the clinical examination in patients with heart failure. JACC Heart Fail. 2018 Jul;6(7):543-51.

26 Murad K, Goff DC Jr, Morgan TM, Burke GL, Bartz TM, Kizer JR, et al. Burden of comorbidities and functional and cognitive impairments in elderly patients at the initial diagnosis of heart failure and their impact on total mortality: the Cardiovascular Health Study. JACC Heart Fail. 2015 Jul;3(7):542-50.

27 Tokitsu T, Yamamoto E, Oike F, Hirata Y, Tsujita K, Yamamuro M, et al. Clinical significance of brachial-ankle pulse-wave velocity in patients with heart failure with preserved left ventricular ejection fraction. J Hypertens. 2018 Mar;36(3):560-8.

28 Smitson CC, Scherzer R, Shlipak MG, Psaty BM, Newman AB, Sarnak MJ, et al. Association of blood pressure trajectory with mortality, incident cardiovascular disease, and heart failure in the Cardiovascular Health Study. Am J Hypertens. 2017 Jun;30(6):587-93.

29 Sacco SJ, Leahey TM, Park CL. Meaning-making and quality of life in heart failure interventions: a systematic review. Qual Life Res. 2019 Mar;28(3):557-65. 
Gao et al.: WHR and HF with Mid-Range Ejection Fraction

30 Wan J, Liu S, Yang Y, Wang D, Ran F, Xia S, et al. Roles of arterial pressure volume index and arterial velocity pulse index trajectories in risk prediction in hypertensive patients with heart failure with preserved ejection fraction. Clin Exp Hypertens. 2020 Jul;42(5):469-78.

31 Chen S, Li W, Jin C, Vaidya A, Gao J, Yang H, et al. Resting heart rate trajectory pattern predicts arterial stiffness in a community-based Chinese cohort. Arterioscler Thromb Vasc Biol. 2017 Feb;37(2):359-64.

32 Ravindrarajah R, Hazra NC, Hamada S, Charlton J, Jackson SH, Dregan A, et al. Systolic blood pressure trajectory, frailty, and all-cause mortality $>80$ years of age: cohort study using electronic health records. Circulation. 2017 Jun;135(24):2357-68.

33 Xu W, Zhang H, Paillard-Borg S, Zhu H, Qi X, Rizzuto D. Prevalence of overweight and obesity among Chinese adults: role of adiposity indicators and age. Obes Facts. 2016;9(1):17-28.

34 He Z, Rankinen T, Leon AS, Skinner JS, Tchernof A, Bouchard C. Plasma steroids, body composition, and fat distribution: effects of age, sex, and exercise training. Int J Obes. 2018 Jul;42(7):1366-77.

35 Song M, Hu FB, Wu K, Must A, Chan AT, Willett WC, et al. Trajectory of body shape in early and middle life and all cause and cause specific mortality: results from two prospective US cohort studies. BMJ. 2016 May; 353:i2195.

36 Xin Y, Chen X, Zhao Y, Hu W. The impact of heart rate on patients diagnosed with heart failure with mid-range ejection fraction. Anatol J Cardiol. 2019 Feb;21(2):68-74.

37 Özlek B, Özlek E, Ağuș HZ, Tekinalp M, Kahraman S, Çil C, et al. Patients with HFpEF and HFmrEF have different clinical characteristics in Turkey: a multicenter observational study. Eur J Intern Med. 2019 Mar;61:88-95.

38 van Woerden G, Gorter TM, Westenbrink BD, Willems TP, van Veldhuisen DJ, Rienstra M. Epicardial fat in heart failure patients with mid-range and preserved ejection fraction. Eur J Heart Fail. 2018 Nov;20(11):1559-66.

39 Packer M. Epicardial adipose tissue may mediate deleterious effects of obesity and inflammation on the myocardium. J Am Coll Cardiol. 2018 May;71(20):2360-72.

40 Moazzami K, Lima BB, Sullivan S, Shah A, Bremner JD, Vaccarino V. Independent and joint association of obesity and metabolic syndrome with depression and inflammation. Health Psychol. 2019 Jul;38(7):586-95.

41 Sutin AR, Boutelle K, Czajkowski SM, Epel ES, Green PA, Hunter CM, et al. Accumulating Data to Optimally Predict Obesity Treatment (ADOPT) core measures: psychosocial domain. Obesity (Silver Spring). 2018 Apr; 26 Suppl 2:S45-54.

42 Cuevas AG, Chen R, Thurber KA, Slopen N, Williams DR. Psychosocial stress and overweight and obesity: findings from the Chicago Community Adult Health Study. Ann Behav Med. 2019 Oct;53(11):NP.

43 Geiker NR, Astrup A, Hjorth MF, Sjödin A, Pijls L, Markus CR. Does stress influence sleep patterns, food intake, weight gain, abdominal obesity and weight loss interventions and vice versa? Obes Rev. 2018 Jan;19(1): 81-97. 